\title{
Ionization and temperature structure in nova shells
}

\author{
H. Beck ${ }^{1}$, H.-P. Gail ${ }^{2}$, H. Gass ${ }^{1}$, E. Sedlmayr ${ }^{1}$ \\ 1 Technische Universität Berlin, Institut für Astronomie und Astrophysik, PN 8-1, Hardenberstraße 36, \\ D-1000 Berlin 12 \\ 2 Institut für Theoretische Astrophysik, Universität Heidelberg, Im Neuenheimer Feld 561, \\ D-6900 Heidelberg 1
}

\begin{abstract}
It is well known from observations that some novae show infrared continuum excesses indicating dust heating by the strong ultraviolet radiation field. It seems, however, that dust formation in nova shells depends on some yet unknown intrinsic parameters since the characteristic infrared excesses are not observed in all objects. To study the conditions under which dust nucleation may occur in nova shells, as a first step we investigate the time dependend ionization and temperature structure.
\end{abstract}

\section{Model calculations}

In our model we consider a spherically expanding shell of constant thickness. The gas is exposed to a diluted black body radiation field with variable effective temperature of the central object. We solve the ionization and recombination rate equations for the elements $\mathrm{H}, \mathrm{He}, \mathrm{C}, \mathrm{N}, \mathrm{O}, \mathrm{Na}, \mathrm{Fe}, \mathrm{Si}, \mathrm{Mg}, \mathrm{S}$ simultaneously with the energy balance equation for calculating the temperature in the gas. Within the expanding spheres of ionizing atoms we have considered photoionization as heating mechanism, and recombination, hydrodynamic expansion, free-free radiation of hydrogen and forbidden line emission from collisionally excited OI, OII, NII, SiII and SII as cooling processes.

\section{Results}

The ionization degrees of the elements H, C and Si at day 26 are shown in Fig.1a and 1b. We have calculated models for two sets of parameters derived from observational data for Nova Muscae 1983 (Case A, Fig. 1a) and Nova Hercules 1934 (Case B, Fig. 1b) which are used as examples for non-dust forming (A) and dust forming (B) novae. The propagation of the $\mathrm{C}$-ionization front is shown in Figs. $2 \mathrm{a}, 2 \mathrm{~b}$ for several instants.

The temperature stratification and the distribution of the electron density in Case $B$ are presented in Fig. 3. The increase of the stellar temperature and the mean temperatures in the HII, CII, and CI region for Case B are given in Fig. 4. The computations show that for the model parameters in Case A the ionization front reaches the outer edge of the shell within about 10 days, thus preventing the occurence of dust nucleation. In Case B, model calculations show that the carbon ionization front only slowly moves through the ejected gas and, thus, for at least 50 days after the outburst there exists an outer shell of neutral carbon atoms. In this zone, the temperature already in an early phase of the expansion drops below $1000 \mathrm{~K}$ although this value depends sensitively on $\mathrm{C}$ abundance, and chemical reactions become possible soon after the outburst. The zone of neutral carbon exists sufficiently long that dust formation can occur at about day 10-100 after the outcast in accord with observations. 

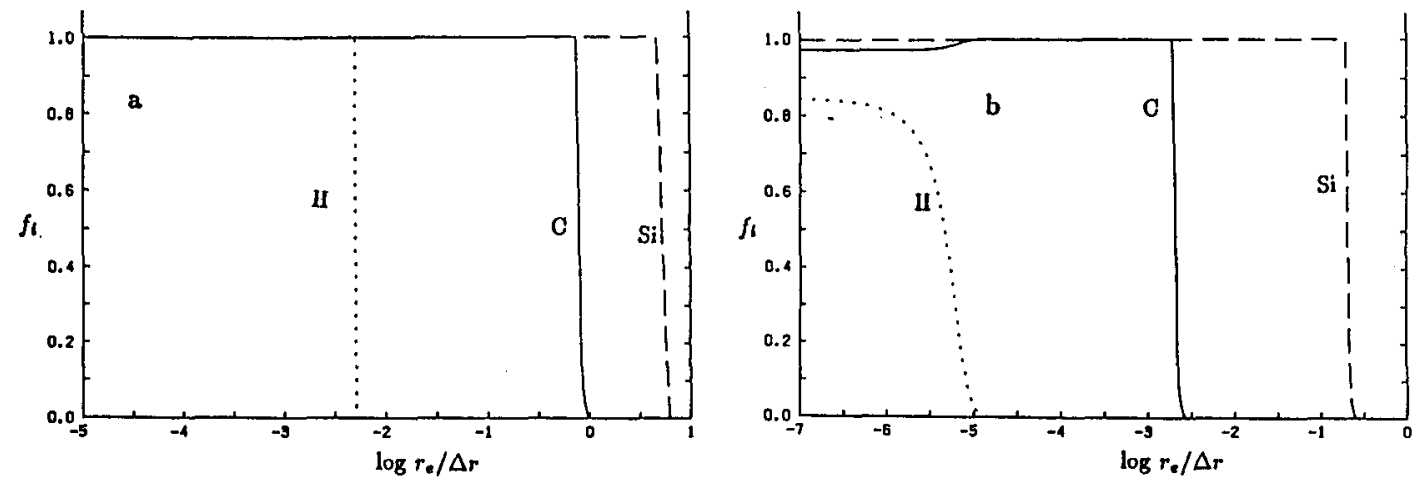

Fig. 1. The ionization structure for non-dust forming (a) and dust forming (b) nova shells is shown for $\mathrm{H}, \mathrm{C}, \mathrm{Si}$ at day 26 after the outcast. The degrees of ionization are plotted versus a logarithmic normalized radius scale, where $r_{e}$ is the distance within and $\Delta r$ the thickness of the shell.
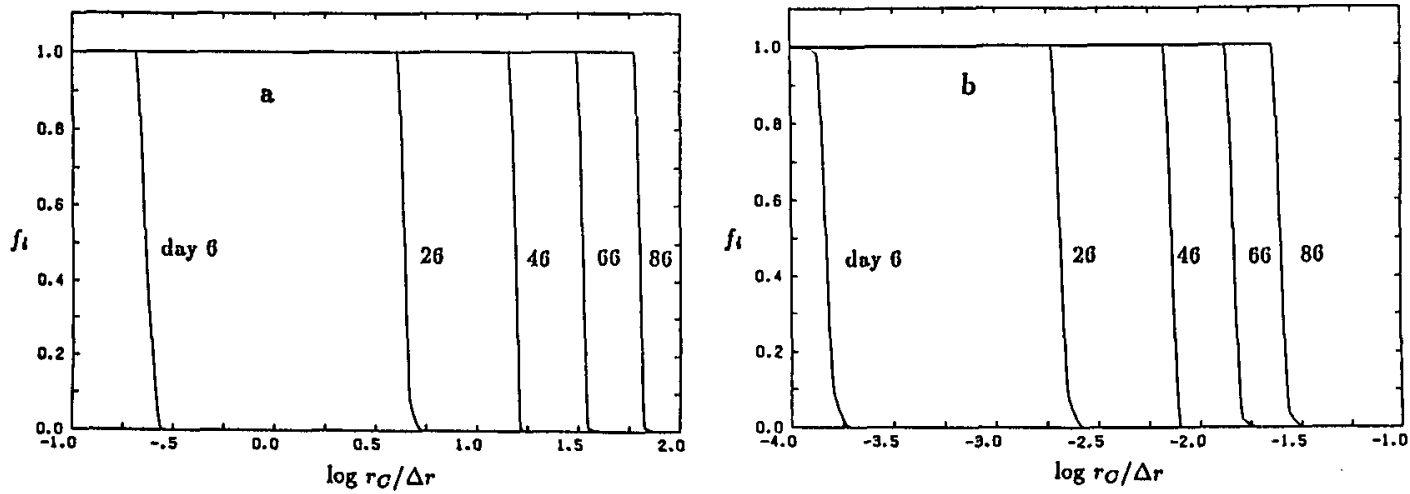

Fig. 2. The propagation of the $C$ ionization front for several days in Case $A$ (a) and Case B (b). The carbon Strömgren radius is denoted by $\mathbf{r}_{\sigma}$.

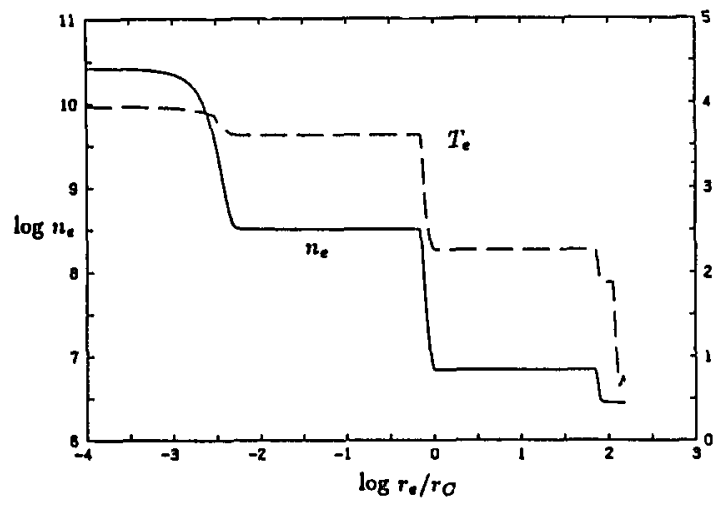

Fig. 3. Electron density and electron temperature at day 26.

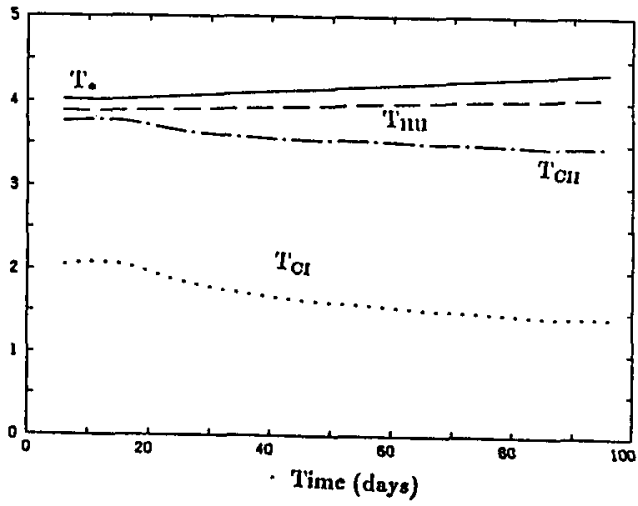

Fig. 4. Stcllar temperature and mean electron temperatures in the HII, CII, and CI region for Case $\mathrm{B}$. 